\title{
Renewable-integrated Traffic Energy
}

\author{
A.K. Dey, JVR Nickey and Y. Sun \\ ${ }^{1}$ Process Energy and Environmental Technology Station, University of Johannesburg, South Africa \\ ${ }^{2}$ Electrical \& Electronics Department, University of Johannesburg, South Africa
}

\begin{abstract}
This work is a development of an indigenous technology combined Flap-motor power generator (FMPG) and PV system that harnesses the free renewable energies in rural area to generate electricity. FMPG and solar renewable energy power technologies are affordable, clean and sustainable and can replace or supplement power generator for road traffic signal light. Combined energy systems integrate these renewable energy technologies with flap base car passing power generators, PV and batteries to provide road signal power in remote areas not connected to a utility grid. Such an isolated grid will help to supply electricity for traffic signal to avoid road accident and maximum vehicle efficiency at intersections. This power generation device will provide constant power supply while no sunlight for long days. At the same time technology will represent instance power supply for rural area traffic light electrification system without grid connection.
\end{abstract}

\section{Introduction}

In this paper we discover technology to generate electricity from car passing simple methodology in which the system used is reliable and this technique will help conserve our natural resources. We got to save the power gained from the conventional sources for efficient use. A multifaceted and cost effective maximum power generation technique is utilized for the motor elevator machine and photovoltaic. The systems utilize flying wheel base permanent magnet motor, which convert mechanical stress into an electrical current. Such materials will be efficient and cost effective with PV solar combined energy source. When cars drive over flaps and temporarily pushed down. When push the flap, permanent magnet motor is forced to rotate through flying wheel and generating electricity. In lower-traffic areas, PV solar panel placed parallel with the system could be used to generate more electricity from combined energy source [1]. This way, the passing of the car system and PV solar combined system would generate electricity with more efficient way and this principle could be implemented in places like the rural area.

\section{Framework}

The main frame model consists of this article is that permanent magnet motor output and PV solar power productivity. When car is passing over flap translation motion produce. It is a device, which converts mechanical energy into electrical energy. The generator uses rotating coils of wire and magnetic fields to convert mechanical rotation into a pulsing direct electric current through Faraday's law of electromagnetic induction. The crank is attached to a system which contains Gearbox, Flywheel \& permanent magnet motor (PMM). For the storing purpose, a Battery is attached to the output of permanent magnet motor. Traffic light input terminal is connect with the Battery to get continuous power source and PV output will support for crises situation like low power source conditions [2]. A cycle reader circuit is placed in front of Flywheel. Logic units are install for the purpose of controlling system. First one is attached between PMM \& Battery and second is between Battery and control unit that break the connection. The third logic unit is placed between the control unit and PV solar power output which indicates the completion of procedure.

\section{Output estimation}

The input-output linearization method is used for obtaining a linear relationship between a input (dw/dt) and the output. Fig. 1 shows the driven FMPG with flying wheel. In this figure; $K_{f}, B_{f}, J_{f}, w_{f}, J_{t}, K_{t}$ and $w_{t}$ are the shaft damping, torsion of the shaft, the rotor inertia, the rotor angular seed, the inertia, the external damping and the angular speed of the FMPG generator, respectively. Also, $T_{f}$ is the aerodynamic torque, $T_{t}$ the high speed shaft torque and $\eta_{t}$ is the gear-belt friction coefficient. The dynamic equations for the low speed shaft and the high speed shaft are:

$T_{f}=K_{f}\left(w_{f}-w_{t}\right)+B_{f}\left(\theta_{f}-\theta_{t}\right) \quad$ And $T_{t}=T_{f} \cdot \eta_{t}$ 
Relationship between the rotor angular speed $\left(w_{f}\right)$ and the electromagnetic torques can be written as:

$$
\mathrm{J} \frac{d w_{f}}{d t}+\left(K_{f}+K_{t} \eta_{t}^{2}\right) w_{f}=T_{f}-T_{t}
$$

The additional input $(\mathrm{dw} / \mathrm{dt})$ is an estimation of the FMPG turbine torque; Input is express as:

$$
\frac{d w}{d t}=\frac{T_{f}-T_{t}}{J}+\frac{K_{f}+K_{t} \eta_{t}^{2}}{J} w_{f}
$$

Thus, the FMPG generator torque is written:

$$
T_{t}=\mathrm{J}\left(\frac{T_{f}}{J}-\frac{d w}{d t}\right)-\left(K_{f}+K_{t} \eta_{t}^{2}\right) w_{f}
$$

So total power developed form FMPG generator is:

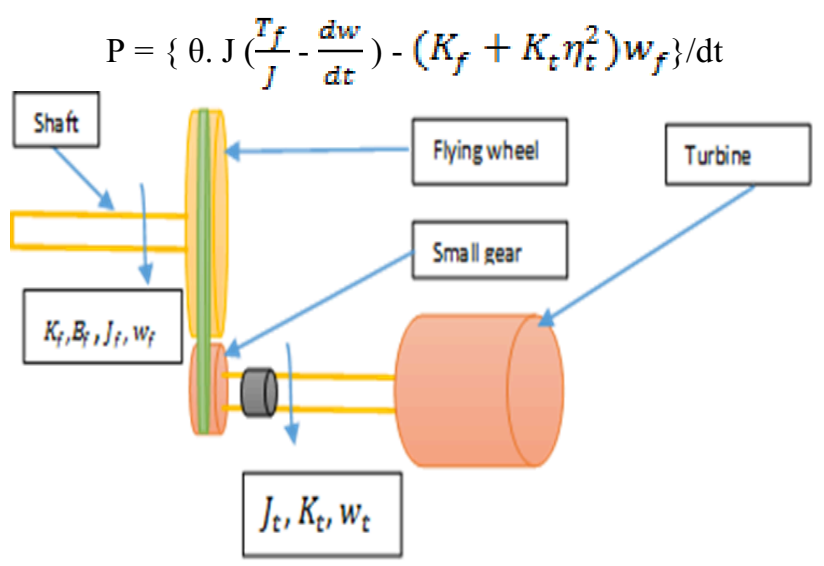

Figure 1. Torque structure of the FMPG system

Flying wheel turning value is the most critical data needed to evaluate the power potential of car passing due to its cubic relation with the power. Car passing on the flap is always variable depends on traffic. Since car passing density varies it is necessary to capture this variation in the model used to predict energy production. This is usually done using probability functions to describe flying wheel number of turn over a period of time. Where $\mathrm{N}=$ number of turning of the wheel, $\xi=$ parameter in turning per second and $\mathrm{k}=$ constant in terms of dimension.

$$
\mathrm{f}(N)=\frac{k}{\eta}[N / \xi]^{k-1} e^{-(N / \xi)^{k}}
$$

The dimension factor defined as: $2 \frac{\pi}{n}$ (weere $n$ is number of rotation).

The output power of the FMPG turbine is related to the speed of flying wheel, and it can be calculated by:

$$
\left\{\begin{array}{c}
0 \\
\frac{\left[v^{a}(t)-v_{c i}^{a}\right] p_{T}}{v_{r}^{s}-v_{c i}^{a}} \\
0
\end{array}\right.
$$

$$
\begin{aligned}
& P_{F M P G}(\mathrm{t})= v_{c i}>v(t) \\
& v_{c i}<v(t)<v_{c o} \\
& v(t)>v_{c o}
\end{aligned}
$$

Where $p_{r}$ is the rated power generator of the FMPG turbine (watt). $\mathrm{V}(\mathrm{t})$ is the variable speed of the flying wheel, $v_{c i}=$ cut-in speed of the flying wheel, $v_{c o}=$ cut- out speed of the flying wheel and $v_{r}=$ rated speed of the turbine.

Thus FMPG generation introduces uncertainty also in operating a power system and it is continuously variable and difficult to predict. Since FMPG power varies randomly there must be a stand source to meet load demand. Energy storage system makes the hybrid system more reliable and affordable. The voltage of the battery bank can be kept lower as compared to the reference dclink voltage ( $V$ dc-ref) by using solar power and receive output pulses $\left(P_{A} \& P_{B}\right)$. In the proposed standalone system, the batteries bank depth of discharge is considered $70 \%$ and is based on the assumption that it should provide the electric power to the loads of a $1.0 \mathrm{~kW}$ for approximately an hour when the generated FMPG power is zero [3].

The battery voltage, $V_{\text {battery }}(\mathrm{t})$, reflects the accumulated energy at the current time $t$ and is calculated in accordance with the simplified model on the basis of the following equation:

$$
\begin{gathered}
V_{\text {battery }}(\mathrm{t})=V_{F M P G}+ \\
\frac{V_{P V}+V_{F M P G}}{W_{\text {bat }-\max }-w_{\text {batt-min }}}\left[w_{F E D}-w_{\text {batt-min }}\right]
\end{gathered}
$$

Where $w_{F E D}$ is the energy fed to the input, and $V_{P V}$ and $V_{F M P G}$ are the voltage value for the maximum energy from PV solar and FMPG system respectively. $W_{\text {batt-max }}$ and $W_{\text {batt-min }}$ the minimum and minimum energy accumulated into the battery, respectively.

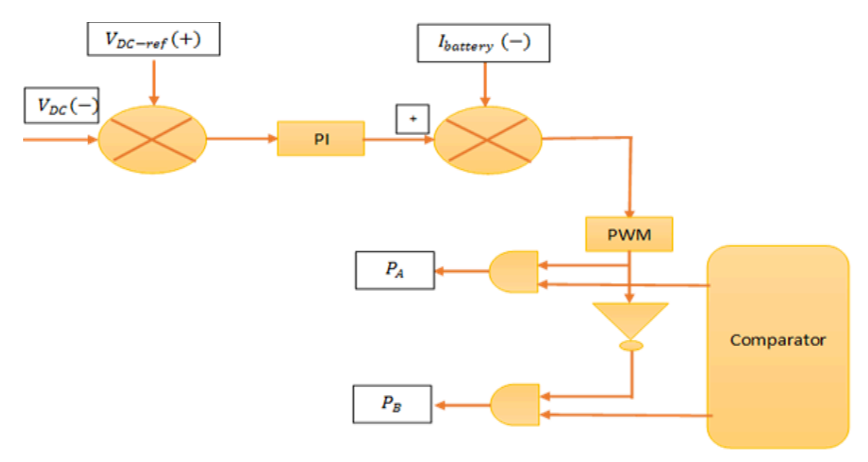

Figure 2. Voltage reference of battery with comparator.

\section{Energy Estimation}

The FMPG/Solar combined model block diagram is shown in the figure below:

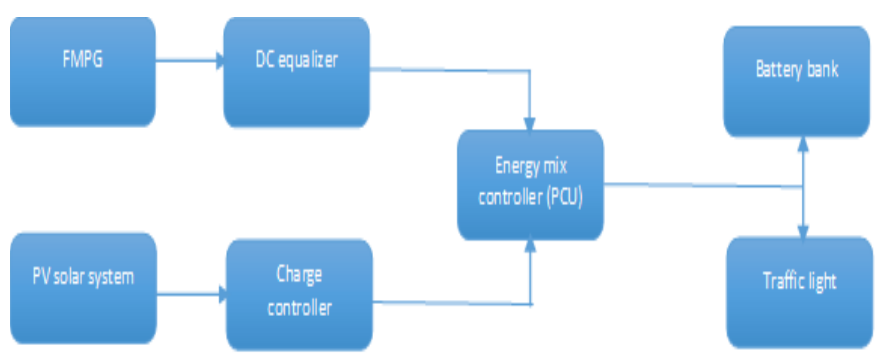

Figure 3. Power combined diagram from different source.

-Flap motor power generator=FMPG. 
-Photovoltaic power generator=PPG.

-Power controlling unit=PCU.

-Battery bank=BAT.

Total power generate from combined system per day:

$$
\begin{aligned}
& P_{\text {total }}(\mathrm{t})=P_{\text {mepg }}(\mathrm{t}) \times N_{\text {car passing }}+ \\
& \mathrm{P}\left(N_{\text {PV module with watt }} \times H_{P_{\text {er day }}}\right)
\end{aligned}
$$

Thus power generation introduces uncertainty also in operating a power system and it is continuously variable and difficult to predict. FMPF power source varies randomly there must be a stand source to meet load demand.

\section{Energy optimization methodology}

The structure of the model developed consists of a database that serves as a backhand tool during the computation and in the front end, calculations on size of the solar, battery and quantity of car passing systems are carried out following an iterative scheme based on the respective average daily data on energy generated. Initially, the efficiency of power generator machines required to meet the average daily demand is calculated on a daily basis throughout a year from which the maximum and minimum quantity of the car passing required is determined [4]. For each size of the generator, the size of solar panel is determined for the difference in load on a daily average basis, from which the maximum and minimum size of the PV panel required. Thus for each size of the generator and solar PV system combination, the maximum and minimum size of the battery are calculated. Innermost loop for every assigned size of the motor elevator machine and PV system is then operated between the minimum and maximum size of the chosen battery model. The optimization of combined systems are carried out in order to minimize the deficiency as well as the excess power generated thereby reducing the cost of the system. However matching the load requirements for a most probable period throughout the year is difficult in most of the cases. Therefore in the present analysis, battery banks are designed/optimized taking into account the deficiency in power generated throughout the year, during the combined operation of power generator and PV system. Constructed on the proposed control system algorithm, the closed-loop system could be exemplified by the equation below. Energy flows from the PV panel, permanent magnet generator and the battery are dispatched by the proposed control system, based on the information of total load consumption. The inclined line implies that the real-time information of energy consumption is fed-back to control system for decision making [5].

The hourly energy production from the PV array of a particular zone:

$$
P V_{p}=g_{P V} \emptyset_{P V} \varepsilon_{P V}
$$

Where $\emptyset_{p V}$ the area of the PV array, $\Omega_{p V}$ is the hourly solar radiation occurrence on the $\mathrm{PV}$ array and $\varepsilon_{p V}$ the PV array efficiency.
As we discussed the power production of the kinetic turbine depends on the how many car passing through the flap [6]. The mathematical model used to convert hourly power generation by the permanent magnate as follows:

$$
\begin{aligned}
& P_{P M}=\varepsilon_{P m} C_{p} T_{a v} \mathrm{~T}_{p m} \\
& \varepsilon_{P m}=\text { Permanent magnet generator efficiency }
\end{aligned}
$$
according to winding of coil.

$\mathrm{C}_{p}=$ Power coefficient of permanent magnet generator.

$T_{a v}=$ average time period of car passing, where $T_{a v}=\left(t_{1}+t_{2} \ldots \ldots t_{n}\right) / 2$.

$\mathrm{T}_{p m}=$ Torque generated by the car passing.

So total power generated by the system:

$$
P V_{p}+P_{P M}=\rho_{p V} \emptyset_{p V} \varepsilon_{p V}+E_{p m} C_{p} T_{a v} \mathrm{~T}_{p m}
$$

Energy flow from the PV and PMG:

where in each time $\mathrm{k}$.

$$
\mathrm{P} \sum(\mathrm{k})=P_{L}(\mathrm{k})-P_{P V}(\mathrm{k})-P_{P M}(\mathrm{k})
$$

The control sequence $\mathrm{u}(\mathrm{k})$ is calculated by using optimal control technique.

$$
\mathrm{u}(\mathrm{k})=\left[P_{P V}(k), P_{P M}(k)\right]^{T}
$$

Transformation process can be create by considering of the dynamic model at the specific system. Characters for the PV system logic controls functioncreate_H ( )

$\{\mathrm{H}=[]$;

$\operatorname{var} \mathrm{p}=2$;

for $(\operatorname{vari}=0 ; \mathrm{i}<=100 ; \mathrm{I}++)\{\mathrm{H}[\mathrm{i}]=\mathrm{i} ; \ldots . .$.

Characters for the permanent magnet turbine power system logic controls functioncompute_pw (compute value of coefficients)

The function compute the output power pw

$\{a=$ compute_a (value of coefficients);

$\mathrm{b}=$ compute $\mathrm{b}$ (value of coefficients); .....

We modelled a PV \& permanent magnet turbine power system integrated in a combined network capturing the system's requirements, the system's structure in terms of the relationship between the different elements, the system's behaviour in terms of activities in the different cases considered and transition from one state to another as well as the system's interaction.

The battery serves as an energy source entity when discharging and a load when charging. At any time, $t$, the state of battery is related to the previous state of charge and to the energy production and consumption situation of the system during the time from $t$ to $\mathrm{t}+1$. On the other hand, when the load demand is greater than the available energy generated, the battery bank is in discharging state. In combined arrangement with PV, FMPG and battery the power generate from PV and the load demand at given a time $t$, determine the charge and discharge power into and out of the battery bank. $\mathrm{T}$ is the integer representing the $t^{\text {th }}$ time interval. The charge status of 
the of the battery bank at the following hour $C_{b}(\mathrm{t}+1)$. The following conditions need to be taken in to consideration for energy flows from $t$ to $(t+1)$, at any given time the battery charge status will be expression by:

$$
C_{b}(\mathrm{t}+1)=C_{b}(\mathrm{t})+E_{c}\left[p_{F}(\mathrm{t})+P_{P V}(t)\right]-P_{d} / E_{d}
$$

Where $\varepsilon_{c} \& \varepsilon_{d}$ is a charge efficacy and discharge efficiency of the battery respectively. $\left(P_{F}+P_{P V}\right) \& P_{d}$ is power acceptance (by FMPG and PV) and discharge by the battery at each time interval.

Similarly for the charging process

$$
C_{b}(\mathrm{t}+1)=C_{b}(\mathrm{t}) \cdot\left(1-S_{d}(\mathrm{t})\right)-\frac{I_{c / d} \varepsilon_{c}}{B_{c}}
$$

Where $I_{c / d}=$ charge and discharge current.

$E_{c}=$ Charge efficiency of the battery

$S_{d}(\mathrm{t})=$ Self discharged rate.

$B_{c}=$ charge capacity of the battery.

$I_{c / d}=$ charge or discharge current.

The available battery bank capacity must not be less than the minimum allowable capacity of $C_{b}(\mathrm{t}+1)$.

Stand-alone FMPG/PV hybrid systems are designed to be totally self-sufficient in generating, storing, and supplying electricity to the traffic light loads in remote areas. The following equation shows the energy balance of a PV/FMPG hybrid system:

$$
E_{F M P G . I N}+E_{P V . I N} \approx E_{F M P G \text { out }}+E_{P V \text { out }}
$$

For the PV part of the hybrid system, device losses include PV charge controller losses, DC-AC conversion losses, both for energy flowing directly to the load and for energy transiting through the battery, and storage round-trip losses. On the turbine side, the AC-DC conversion losses affect electrical energy that does not flow directly to the load. The reason for these losses on the generator side is that the hybrid system was designed to be cycle charging meaning that the turbine is allowed to charge the battery. The energy that has to be delivered from the FMPG can be express as:

$$
E_{F M P G}=E_{\text {load }}+E_{\text {loss }}+E_{R \text { loss }}-E_{P V}
$$

The energy that has to be delivered from the photovoltaic can be express as

$$
E_{P V}=E_{\text {load }}+E_{\text {loss }}+E_{B \text { loss }}-E_{F M P G}
$$

Where $E_{R \text { loss }} \& E_{B \text { loss }}$ are rectifier loss and battery loss respectively.

The turbine generator should be operated near its nominal power to achieve high energy efficiency by the inclusion of battery bank. As shown in above equations, energy losses are flowing into the energy demand and supply of the system; therefore, it is necessary to identify the energy losses in the system. System losses define systematic energy losses that are due to the physical properties of the system components or the entire installation. Energy conversion losses constitute important contributions to this hybrid system.

\section{Conclusion}

This paper investigates the designing of a stand-alone hybrid power system focusing on photovoltaic/FMPG energy system with energy storage in batteries. Starting from the analysis of the models of the system components, a complete model is realized. Renewable independent systems cannot provide continuous source of energy, as they are seasonal. By integrating and optimizing the solar photovoltaic and motor-flap power generator systems, the reliability of the systems can be improved and the unit cost of power can be minimized. A primary model was developed to optimize combine system for any specific location, by considering the parameters deficiency of power system. The optimal size of the combined system is determined based on the calculated values of actual power consumption for traffic light. Thus the model suggests the optimum combination of the capacity of motor elevator power generator, PV and battery units of a chosen type that can generate power with a minimum excess power generation by implementation of iterative technique.

\section{Reference}

1. Xiongwen Zhang, Siew-Chong Tan, Guojun Li, Jun $\mathrm{Li}$, and Zhenping Feng. Components sizing of hybrid energy systems via the optimization of power dispatch simulations. Energy, Page: 165-172, April 2013.

2. B. Tudu, S. Majumder, K.K. Mandal, and N. Chakraborty. Optimal unit sizing of stand-alone renewable hybrid energy system using bees algorithm. 2011 International Conference on Energy, Automation and Signal, pages 1-6, December 2011.

3. R. Luna-Rubio, M. Trejo-Perea, D. Vargas-V'azquez, and G.J. R'10s-Moreno. Optimal sizing of renewable hybrids energy systems: A review of methodologies. Solar Energy, page: 1077-1088, April 2012.

4. Ajai Gupta, R. P. Saini, and M. P. Sharma. Computerized modelling of hybrid energy system Part II: Combined dispatch strategies and solution algorithm. In 2008 International Conference on Electrical and Computer Engineering, pages 13-18. IEEE, 2008.

5. S. Farahat, M.A. Yazdanpanah Jahromi, and S.M. Barakati. Modeling and Sizing Optimization of Stand-alone Hybrid Renewable Energy Systems. International Conference on Mechanical, Nanotechnology and Cryogenics Engineering, pages 212-217, 2012.

6. Center for Energy Efficiency \& Renewable Energy. The Hybrid Power System Simulation Model, 2014. 